\title{
Detection of Entamoeba histolytica/Entamoeba dispar in Stool Specimens by Using Enzyme-linked Immunosorbent Assay
}

\author{
Nuran Delialioglu/ ${ }^{+}$, Gonul Aslan, Mehmet Sozen, Cahit Babur*, Arzu Kanik**, \\ Gurol Emekdas
}

\author{
Department of Microbiology and Clinical Microbiology *Refik Saydam Hygiene Center, Ankara, Turkey \\ **Department of Biostatistics, Faculty of Medicine, Mersin University, Mersin, Turkey
}

Entamoeba histolytica actually comprises two genetically distinct but morphologically indistinguishable species. E. histolytica can cause invasive intestinal and extra intestinal disease, while $\mathrm{E}$. dispar cannot. Identification and differentiation of $\mathrm{E}$. dispar and $\mathrm{E}$. histolytica in stool sample by microscopy is imprecise. Several weeks of culture and isoenzyme analysis are required to differentiate $\mathrm{E}$. histolytica from $\mathrm{E}$. dispar. In this study, we have used an enzyme-linked immunosorbent assay (ELISA) for detection of E. histolytica/E.dispar and compared it with microscopy. Eighty-eight samples were evaluated, trichrome staining was positive in $20.4 \%$ (18) and ELISA was positive in $29.5 \%$ (26). Both tests were positive in 14 (15.9\%) samples, 4 (4.5\%) only with direct microscopy, and $12(13.6 \%)$ only with ELISA. Both tests were negative in 58 (65.9\%) samples. Microscopy has low sensitivity and high specificity, low negative predictive value and high positive predictive value in comparison with ELISA.

E. histolytica/E. dispar antigen detection ELISA tests are inexpensive compared to the specific tests, yield objective results and do not require experienced microscopists and can therefore be recommended for screening of stools worldwide and the results can be taken for treatment that are fitting with its clinic.

Key words: Entamoeba histolytica/E. dispar antigen - microscopy - ELISA

Amoebiasis is one of the most common parasitic infections worldwide (Trol et al. 1997, Haque et al. 1998). Entamoeba histolytica can be found worldwide but is more prevalent in tropical and subtropical regions. Humans are the primary reservoir; infection occurs by ingestion of cysts from fecally contaminated material such as water and food (Leber \& Novak 1999). Although about 500 million people each year have amoebiasis, only about $10 \%$ experience symptomatic disease (Trol et al. 1997, Haque et al. 1998). An estimated 40,000-100,000 people die yearly from amoebiasis, this disease is the second leading cause of death from parasitic disease worldwide (Stanley 2003). Concept of the existence of two morphologically identical amoeba species was formulated by Emile Brumpt in 1925. Since there was no way of distinguishing between the cysts of pathogenic and non-pathogenic species, Brumpt's suggestion was not accepted (Myjak et al. 2000, Ackers 2002). Sargeaunt and Williams have demonstrated that there were differences between isoenzymes of pathogenic and non-pathogenic isolates in 1988. A lot of biochemical, immunological and genomic differences between the two species were recognized and led to formal separation of the two species with the name $E$. histolytica being retained pathogenic species and Brumpt's name E. dispar being revived for the non-pathogen (Ackers 2002).

${ }^{+}$Corresponding author. Fax: +90.324.3374305. E-mail: nurandel@hotmail.com

Received 29 March 2004

Accepted 17 September 2004
It is generally accepted that what was earlier known as E. histolytica actually comprises two genetically distinct but morphologically indistinguishable species. $E$. histolytica can cause invasive intestinal and extra intestinal disease, while E. dispar cannot. Identification and differentiation of E. dispar and E. histolytica in stool sample by microscopy is imprecise. Not only is microscopy unable to differentiate E. histolytica from E. dispar, can be founded by false-positive results due to misidentification of macrophages and nonpathogenic species of Entamoeba. After many studies, it is obvious that culture is more sensitive than microscopy, and isoenzyme analysis of cultured amoebae enables the differentiation of $E$. histolytica from E. dispar. Amoebic cultures and isoenzyme analysis require a week to complete and are negative in many microscopy positive samples due to delays in sample processing or due to the probable antiamebic therapy before stool collection. Detection of E. histolytica by using ELISA, enzyme-linked immunosorbent assaybased stool antigen detection kits and detection of $E$. histolytica specific DNA by polimerase chain reaction (PCR) amplification can be done. Antigen detection tests have proven to be more sensitive and specific than microscopy (Haque et al. 1998).

In this study, we have used an ELISA for detection of E. histolytica/E.dispar and compared it with microscopy.

\section{MATERIALS AND METHODS}

Stool specimens were collected from patients with blood and/or mucus diarrhea on March 2003 and September 2003 at the Mersin University, Faculty of Medicine and Mersin Social Insurance Institution Hospitals, Mersin. Eighty-eight stool specimens were collected from 40 child patients between 2-14 ages and 48 adult patients between 16-50 ages; 88 stool specimens were trichrome stained 
and examined by microscopy. E. histolytica/E. dispar antigen (Ridascreen Entamoeba; R-Biopharm AG, Darmstadt, Germany) detection was performed by an enzyme immunoassay method. The detected antigen type during the ELISA's reaction was E. histolytica sensu lato. The entamoeba test is an in vitro diagnosis intended for the detection of E. histolytica/E. dispar in fecal specimens. Test was performed according to manufacturer's instructions. On the surface of the microtiter wells, monoclonal antibodies against $E$. histolytica sensu lato were bound. Stool specimens were diluted 1:11 in diluents provided with the kit. Assay microtiter wells were incubated with two drops diluted samples and two drops of enzyme conjugate for $60 \mathrm{~min}$ at room temperature. The contents of the well strips were decant and were washed four times in the wash solution (Tektime, bioMerieux). Substrata solution was added and the strip was incubated at room temperature in the dark for $15 \mathrm{~min}$. Following the incubation, the reaction was stopped by adding one drop of stop solution to each well and the absorbance was measured at $450 \mathrm{~nm}$ (Tektime). The assay run was correct, if optical density for the negative control was below 0.2 and the optical density for the positive control was above 0.8 . The cut-off was determined by addition of 0.15 absorbance units to the measured absorption of the negative control.

\section{RESULTS}

Of the 88 samples evaluated, trichrome staining was positive in $20.4 \%$ (18) and ELISA was positive in $29.5 \%$ (26). Both tests were positive in 14 (15.9\%) samples: 4 (4.5\%) only with direct microscopy and $12(13.6 \%)$ only with ELISA. Both tests were negative in $58(65.9 \%)$ samples (Table). The positivity rate in children and adults were $27.5 \%$ (11) and $14.5 \%$ (7) with trichrome staining and 32.5\% (13) and 27\% (13) with ELISA, respectively. In this comparison with ELISA, it was detected that microscopy has $53.8 \%$ sensitivity and $94 \%$ specificity, $78 \%$ positive predictive value and $17 \%$ negative predictive value. Between these two methods, medium degree relation was defined statistically by kappa test (kappa: $0.52, p=0,0001$ ).

The stools of patients in whom microscopic examination was positive were examined macroscopically and $50 \%$ (9) contained blood, 50\% (9) contained blood and mucus, and $100 \%$ (18) contained mucus. These rates were 30\% (21), 27\% (19), and 78.5\% (55), respectively, in patients in whom microscopic examination was negative. Of the patients whose fecal antigen with ELISA was positive, $30.7 \%$ (8) had fecal blood, $30.7 \%$ (8) had blood and mucus, and $69.2 \%$ (18) had mucus. These rates were $24 \%$ (15), $24 \%$

TABLE

Comparison of samples by microscopy and ELISA results

\begin{tabular}{lccc}
\hline & $\begin{array}{c}\text { ELISA } \\
\text { positive } \\
n\end{array}$ & $\begin{array}{c}\text { ELISA } \\
\text { negative } \\
n\end{array}$ & $\begin{array}{c}\text { Total } \\
\text { n }\end{array}$ \\
\hline Microscopy positive & 14 & 4 & 18 \\
Microscopy negative & 12 & 58 & 70 \\
\hline Total & 26 & 62 & 88 \\
\hline
\end{tabular}

(15), and 74\% (46), respectively, in patients whose fecal antigen with ELISA was negative. Erythrocytes were present in the direct microscopic examination of stools in $61 \%$ (11) of patients whose microscopic examination was positive and in $44.2 \%$ (31) of cases whose microscopic examination was negative. Fecal erythrocytes were detected in $46.1 \%$ (12) of patients whose antigen was positive with ELISA and in 45.1\% (28) of patients whose antigen was negative.

On the analysis of microscopy positive and negative samples, existing of blood $(p=0.01)$, blood-mucus $(p=$ $0.063)$ or erythrocyte $(p=0.202)$ have no significance statistically by Chi-Square $\left(\chi^{2}\right)$ test, but existing of mucus ( $\mathrm{p}$ $=0.034)$ has. On the analysis of ELISA positive and negative samples, existing of blood $(p=0.522)$, blood-mucus $(\mathrm{p}=0.522)$ or erythrocyte $(\mathrm{p}=0.932)$ have no significance statistically by $\chi^{2}$ test, but existing of mucus $(\mathrm{p}=0.016)$ has in the same way.

\section{DISCUSSION}

While E. histolytica is the cause of invasive intestinal and extra intestinal infections, E. dispar is non-pathogenic (Haque et al. 1998). The definitive diagnosis of intestinal amoebiasis depends on the history, clinical findings and detection of cysts or trophozoits of the pathogen in the stool. Microscopic examination is based on trichrome or iron hematoxylin staining of fresh stool preparations (Ravdin 2000). However, differentiation of $E$. histolytica and E. dispar is not possible with this method. Some researchers suggest that microscopic examination of the stool is sufficient to diagnose E. histolytica in the presence of characteristic microscopic findings (Stanley 2003). Discussions carried out on this subject by the World Health Organization in Mexico in 1997 yielded the following statements: E. histolytica and E. dispar are morphologically similar and since they cannot be differentiated by light microscopy, they should be reported as $E$. histolytica/E. dispar; asymptomatic cases should not be treated unless $E$. histolytica/E. dispar differentiation is achieved; cases with a definitive diagnosis of $E$. histolytica infection should receive treatment. Sensitive and specific tests such as isoenzyme analyses, antigen detection, hybridization with type specific DNA probes and PCR are available for the detection of E. histolytica in the stool (Haque et al. 1998, Ackers 2002). Antigen detection tests are reported to be more sensitive and specific than direct microscopic examination (Haque et al. 1998). Detection of antigens in the stool and serum is a revolution in the diagnosis of E. histolytica, due to the ease of use, high sensitivity and specificity of available tests (Ravdin 2000). The use of antibody detection tests in serum is recommended in the diagnosis of invasive intestinal and extra intestinal amoebiasis. Anti-amoebic antibodies develop at the end of a week following exposure in symptomatic patients and persist for years (Petri \& Singh 1999, Ravdin 2000).

In a study by Haque et al. (1995), microscopic examination, culture, and antigen detection tests were compared in 202 patients. Considering culture as the gold standard, ELISA was more sensitive and specific than microscopy. In addition, E. histolytica specific ELISA was more rapid 
and reliable (sensitivity $95 \%$ and specificity 93\%) than zymodem analysis in differentiating $E$. dispar from $E$. histolytica in stool samples. On the other hand, Gonin and Trudel (2003) found that ELISA was less sensitive than microscopy and PCR in differentiating $E$. histolytica and $E$. dispar in stool samples. In the same study, it was reported that PCR was more sensitive than ELISA and microscopy, and more specific than ELISA. Haque et al. (1998) compared PCR, izoenzyme analysis and $E$. histolytica-specific antigen detection tests and reported that the sensitivities of PCR and antigen detection tests were $87 \%$ and $85 \%$, respectively. They concluded that the antigen detection test could be used in the diagnosis of E. histolytica infection because it is fast, easy to use and does not require special technical equipment.

The prevalence of infection using antigen detection tests was reported to be $4.7 \%$ in a survey conducted with 680 asymptomatic children aged 2-5 years in Bangladesh (Haque et al. 1999). Schunk et al. (2001), indicate that the E. histolytica/E. dispar EIA test is $81.8 \%$ sensitive and $99.2 \%$ specific compared to microscopy, and it has no cross reaction with other intestinal parasites.

In a study conducted in Ankara, Turkey, E. histolytica/ E. dispar cysts or trophozoits were detected in $13.3 \%$ of 142 mentally retarded individuals and in $9.1 \%$ of 77 stool samples sent for parasitological examination. While the positivity rates in these same samples with E. histolytica/ E. dispar screening ELISA were $19 \%$ and $20.7 \%$, respectively, the rates fell to $0.7 \%$ and $1.3 \%$ when E. histolyticaspecific antigen detection test was used. The true prevalence of E. histolytica was 15-30 times lower with the specific antigen detection test than with microscopy and the screening test because E. histolytica and E. dispar differentiation is not possible with the latter two (Nar et al. 2003). As a result of our study, it is found that microscopy has low sensitivity and high specificity in comparison with ELISA.

The microbiology laboratory records of the Faculty of Medicine, Mersin University during 1999-2000 reveal that the prevalence of E. histolytica/E. dispar cysts was $2.8 \%$ in direct microscopic examination of fresh stool preparations. The prevalence was reported to be $16.5 \%$ in the Mersin State Hospital during the same time period. When the distribution of parasites was analyzed, E. histolytica/ E. dispar was the most frequently detected parasite (Ozturk et al. 2001).

In the present study, the positivity of E. histolytica/ E. dispar was $20.4 \%$ with direct microscopy and $29.5 \%$ with the E. histolytica/E. dispar antigen detection ELISA test in patients presenting with bloody stools and/or blood and mucus in their stools. While ELISA test was positive in 14 of the cases who had E. histolytica/E. dispar cysts in their stools, 4 cases had negative results. On the other hand, 12 samples with a negative result with direct microscopy were positive with the ELISA antigen detection test. This difference may be attributed to the quantity of the pathogen in stools: stools with a low number of cysts, may be negative in the direct microscopic examination and may yield positive results with the ELISA test. In this comparison with ELISA, it is detected that microscopy has $53.8 \%$ sensitivity and $94 \%$ specificity, $78 \%$ positive predictive value and $17 \%$ negative predictive value.

As a result, laboratory examination plays a major role in the diagnosis of intestinal amoebiasis, as well as the history of the patient. Although direct microscopic examination is inexpensive compared to antigen detection tests, the subjectivity of the test, the requirement for experienced microscopists due to the difficulty in differentiating the parasite from leucocytes and other intestinal parasites and its inability to differentiate pathogen species from nonpathogenic forms limits its reliability. Culture, isoenzyme analysis and PCR are time consuming tests. In addition, PCR requires the use of expensive technical equipment. Therefore, these tests are not appropriate for the rapid diagnosis of the disease and prevalence studies, and are used for research. E. histolytica/E. dispar antigen detection ELISA tests are inexpensive compared to the above mentioned tests, yield objective results and do not require experienced microscopists and can therefore be recommended for screening of stools worldwide. However, since these tests cannot differentiate pathogen and nonpathogenic species, positive test results with these tests should be confirmed with the E. histolyticaspecific antigen detection test. Thus, determination of the true prevalence of E. histolytica is possible and unnecessary use of antiparasitic drugs can be prevented.

\section{REFERENCES}

Ackers JP 2002. The diagnostic implications of the separation of Entamoeba histolytica and E. dispar. J Biosc (Supl. 3) 27: 573-578.

Gonin P, Trudel L 2003. Detection and differentiation of Entamoeba histolytica and Enatamoeba dispar isolates in clinical samples by PCR and enzyme-linked immunosorbent assay. J Clin Microbiol 41: 237-241.

Haque R, Ali IKM, Akther S, Petri WA 1998. Comparison of PCR, isoenzyme analysis and antigen detection for diagnosis of Entamoeba histolytica infection. J Clin Microbiol 36: 449-452.

Haque R, Ali IM, Petri WA 1999. Prevalence and immune responce to Entamoeba histolytica infection in preschool children in Bangladesh. Am J Trop Med Hyg 60: 1031-1034.

Haque R, Neville LM, Hahn P, Petri WA 1995. Rapid detection of Entamoeba infection by using Entamoeba and Entamoeba histolytica stool antigen detection kits. J Clin Microbiol 33: 2558-2561.

Leber AL, Novak SM 1999. Intestinal and urogenital amebae, flagellates, and ciliates. In PR Murray, EJ Baron, MA Pfaller, FC Tenover, RH Yolken (eds), Manual of Clinical Microbiology, 7th ed., ASM Press, Washington D.C., p.1391-1405.

Myjak P, Kur J, Pietkiewicz H, Kotlowski A, Nahorski W, Szostakowska B 2000. Molecular differentiation of Entamoeba histolytica and Entamoeba dispar from stool and culture samples obtained from polish citizens infected in tropics and in Poland. Acta Protozool 39: 217-224.

Nar S, Akbas E, Esen B 2003. Diski örneklerinde Entamoeba histolytica ve Entamoeba dispar in arastirilmasinda direkt mikroskobi ve ELISA yöntemlerinin karsilastirilmasi. Flora 8: 213-220.

Ozturk C, Delialioglu N, Aslan G, Aslan N 2001. Mersin bölgesinde barsak parazitlerinin prevalansi ve dagýlýmý; Mersin Üniversitesi ve Devlet Hastanesi Mikrobiyoloji Laboratuvarina ait sonuclar. T Parazitol Derg 25: 355-358. Petri WA, Singh U 1999. Diagnosis and management of amebia- 
sis. Clin Infect Dis 29: 1117-1125.

Ravdin JI, 2000. Entamoeba histolytica (Amebiasis). In GL Mandel, JE Bennett, R Dolin (eds), Principles and Practice of Infectious Diseases, 5th ed., Churchill Livingstone, Philadelphia, p. 2798-2810.

Stanley SL 2003. Amoebiasis. The Lancet 361: 1025-1034.

Schunk M, Jelinek T, Wetzel K, Nothdurft HD 2001. Detec- tion of Giardia lamblia and Entamoeba histolytica in stool samples by two enzyme immunoassays. Eur J Clin Microbiol Infect Dis 20: 389-391.

Trol H, Marti H, Weiss N 1997. Simple differential detection of Entamoeba histolytica and Entamoeba dispar in fresh stool specimens by sodium acetate-acetic acid-formalin concentration and PCR. J Clin Microbiol 35: 1701-1705. 